\title{
The Use of Coding Methods to Estimate the Social Behavior Directed toward Peers and Adults of Preschoolers with ASD in TEACCH, LEAP, and Eclectic "BAU" Classrooms
}

\author{
Ann Sam \\ Frank Porter Graham Child Development Institute, \\ University of North Carolina, Chapel Hill, NC
}

Stephanie Reszka

Department of Allied Health, Division of Occupational Science and Occupational Therapy, University of North Carolina, Chapel Hill, NC

Samuel Odom

Frank Porter Graham Child Development Institute, University of North Carolina, Chapel Hill, NC

Kara Hume

Frank Porter Graham Child Development Institute, University of North Carolina, Chapel Hill, NC

Brian Boyd

Department of Allied Health, Division of Occupational Science and Occupational Therapy, University of North Carolina, Chapel Hill, NC

\begin{abstract}
Momentary time sampling, partial-interval recording, and event coding are observational coding methods commonly used to examine the social and challenging behaviors of children at risk for or with developmental delays or disabilities. Yet there is limited research comparing the accuracy of and relationship between these three coding methods. By coding the low-frequency social behaviors of 100 preschool-aged children with autism spectrum disorder, the current study examines the associations among the three methodologies. Results indicated a strong relationship among all three coding methodologies. The findings provide information to researchers and practitioners considering the benefits and drawbacks of each coding methodology.
\end{abstract}

Various observational coding methods have been employed across research studies in an effort to assess children's social behavior (Odom \& Ogawa, 1992). Determining the best method of capturing and examining social behaviors is of particular interest in research involving children with autism spectrum disorder (ASD). Children with ASD characteristically display low incidences of social behaviors (American Psychiatric Association, 2000; Reszka, Odom, \& Hume, 2012), making direct assessment of these behaviors a challenge (Cunningham, 2012; Tsao et al., 2008). Yet these behaviors are important to capture in order to best identify ecological features of classrooms and interventions that will promote the social development of children with ASD (Reszka et al., 2012). A variety of coding methods have been used to capture lowoccurring behaviors, such as the social behaviors of young children with ASD (Gardenier, MacDonald, \& Green, 2004; Reszka et al., 2012; Tsao et al., 2008); however, no study has directly compared these coding methods in this group of children to determine the utility of each. 
Three commonly used coding methods include momentary time sampling (MTS), partial-interval recording (PIR), and event coding. Event coding, also called frequency counting, is often viewed as the most precise observational method for assessing the quantity of behavior because the observer records each instance of the behavior (Suen \& Ary, 1989). However, due to the intensity of data collection, event coding is sometimes difficult to collect in real time and in field settings. PIR and MTS are designed to estimate the quantity of behavior that occurs and were developed as alternatives to event coding because they may be less difficult to collect. However, these discontinuous methods of observational coding only provide estimates of behaviors because they do not record each behavioral occurrence. MTS involves coding a behavior that only occurs at a specific point in time during a specified time frame (e.g., the observational point in time may occur every $10 \mathrm{~s}$, and only behaviors that occur at that moment in time are coded; Thompson, Symons, \& Felce, 2000). For PIR, in contrast, researchers observe for a period of time (e.g., $10 \mathrm{~s}$ ) and record a behavior as having occurred at any point during the specified time frame (Thompson et al., 2000).

A variety of factors contribute to decisions about the selection of behavior observation and recording systems, including the available resources, the complexity of the coding scheme, and the type of behavior observed. Generally, continuous observation and recording is deemed most desirable by researchers (e.g., event coding); however, this method is often time- and cost-prohibitive. Researchers then must carefully weigh the costs and benefits of other sampling methods (PIR, MTS) and select the most feasible option, perhaps sacrificing perceived accuracy, including overestimating or underestimating behaviors (Gardenier et al., 2004). This notion of reduced or compromised accuracy requires further exploration as the accuracy of outcomes reported in research studies may depend heavily on the type of coding methodology utilized.

In the few studies that have examined the relationships between coding methods, the results indicated that data analyzed using different methodologies are at least moderately correlated. One study of primate social behaviors found that MTS and event coding were moderately correlated ( $r=0.55-0.66)$, and event coding and PIR were more strongly correlated ( $r=0.76-0.96)$ (Rhine \& Linville, 1980). Rhine and Linville recommended using PIR coding rather than MTS because estimates of behavior from PIR coding were more closely related to the actual duration and frequency of behavior occurrence.

Compared to PIR, MTS may be more accurate for observations of the duration of behaviors, but both MTS and PIR provide inaccurate estimates of behavior rates (Harrop \& Daniels, 1986). Harrop and Daniels used computer-simulated data to compare the results of coding the rates and duration of behaviors using MTS and PIR methods. Comparisons between coding methods often are conducted using simulated data (e.g., Harrop, Daniels, \& Foulkes, 1990; Kearns, Edwards, \& Tingstrom, 1990; Rapp, Colby-Dirksen, Michalski, Carroll, \& Lindenberg, 2008). Simulated data refers to data in which responses are generated by a computer rather than by observations of humans or other live organisms. Harrop and Daniels used an observation window of $1 \mathrm{~s}$ out of $15 \mathrm{~s}$ for MTS and observed the behavior for 10-s intervals with a 5-s nonobservation break for PIR over the course of $1 \mathrm{hr}$. The behavior of interest was simulated to occur at constant durations $(1,5,10$, and 20 s) with expected frequencies ranging from 20 to 120 behaviors per hour in the "low to medium" rate group and 120-720 times per hour in the "medium to high" rate group. MTS tended to be less sensitive when detecting changes in the rates and durations of behaviors, and PIR tended to provide estimates with more systematic error when estimating relative changes in behaviors (Harrop \& Daniels, 1986; Powell et al., 1975). PIR, in particular, overestimates the rates of behavior when behaviors occur at lower rates and underestimates when behaviors are short in duration and occur at higher rates (Harrop \& Daniels, 1986).

Repp, Deitz, Boles, Deitz, and Repp (1976) also found that PIR underestimates behaviors that occur at higher rates; however, they noted that estimates of behaviors at low or medium rates were fairly accurate. Using simulated data (generated by electromechanical pulses from pens), they simulated rates of 10 behaviors per minute, 1 per minute, and 0.1 per minute and then of varying patterns of responses (i.e., constant across the session or in bursts of varying rates across the session). In relation to MTS, they found it to underestimate 
behavior responses when responses did not occur at a consistent rate throughout the entire observation as well as when the responses were at medium ( 1 per minute) or high (10 per minute) rates and to overestimate behaviors that occurred at low rates (Repp et al., 1976).

In addition, methodologists propose that for low-frequency behaviors MTS may underestimate the frequency of events that actually occur, which may be better captured using an event recording system (Suen \& Ary, 1989). Ary and Suen (1983) conducted a simulated study to compare the accuracy of MTS and PIR with respect to the duration and frequency of behaviors. They generated data representing $1800 \mathrm{~s}(30 \mathrm{~min})$ of observations with frequencies ranging from 2 to 12 and durations set at 10-15, 30-60, and 90-120 s and simulated 100 cases for each behavioral situation. They found that for shorter observation intervals, the frequency measures were perfectly accurate, and the estimates of duration using MTS were very accurate, with $1 \%-3 \%$ of intervals with errors in estimating duration. Generally, shorter intervals of MTS will provide more accurate estimates of the durations and frequencies of behaviors, but the intervals of observation should be set based on how often the behavior of interest is expected to occur (Ary \& Suen, 1983).

The studies described above all used simulated data. Studies comparing these methods using actual data collected as part of a research study are less common throughout the literature, yet this type of study likely has the most relevance to inform research and practice. It should be noted, however, that observational coding systems have been used within the context of descriptive and intervention studies involving children with ASD although the purpose of these studies was not to compare behavioral observation systems. For example, Yoder and Stone (2006) examined how two interventions ([a] responsive education and prelinguistic milieu teaching [RPMT] and [b] picture exchange communication system [PECS]) impacted the social behavior (i.e., requesting, initiating joint attention, and exchanges) of preschool children with autism by coding frequency of social behaviors. Similarly, frequency data was used to examine the impact of an enhanced milieu teaching intervention to increase social communication skills of preschool children with autism (Hancock \& Kaiser, 2002). Additionally, Wong and Kasari (2012) used continuous recording in 5-min intervals to determine the joint attention and play skills of children with autism in preschool classrooms. Partial interval recording systems also have been employed to examine if an intervention targeting social skills increases these skills in preschoolers with autism (Conroy, Boyd, Asmus, \& Madera, 2007). Finally, several studies have examined ecological features of classrooms and the resulting social behavior of preschoolers with disabilities, including those with autism, using MTS (Reszka et al., 2012; Tsao et al., 2008). Using MTS, Reszka and colleagues found very low incidences of social behaviors during playtime activities in preschool classrooms (less than 2\%). This method may be especially problematic when observing the social behaviors of children with ASD as it may be more likely than for event recording or PIR that social behaviors will be missed in the coding process because of the low frequency of the behavior.

Given difficulties with social interactions, social skill improvements are often a primary target of intervention for children with autism (e.g., Hancock \& Kaiser, 2002; Wong \& Kasari, 2012; Yoder \& Stone, 2006). Due to this, there is a need to determine how to best assess, observationally, these behaviors to obtain the most appropriate, accurate, and reliable data while still being sensitive to the time and cost restraints of research projects.

\section{Research Questions}

This study compares the associations among the three commonly used behavioral observation methods noted previously (event coding, MTS, PIR). The specific research questions in this study are what are the associations among three observational methodologies (event coding, MTS, and PIR) for the social behaviors of children with ASD directed toward adults and peers, and using the three methodologies, are there significant differences in social behaviors for children with ASD enrolled in treatment and education of autistic and related communicationhandicapped children (TEACCH), Learning Experiences: alternative programs for preschoolers and parents (LEAP), and business as usual (BAU) classrooms?

\section{Method}

This analysis was part of a larger study comparing the efficacy of school-based, com- 
prehensive treatment models for preschoolers with ASD (Boyd et al., 2014).

\section{Child Participants}

Participants included 100 preschool-aged children (84 males, 13 females) across four states (North Carolina, Minnesota, Florida, and Colorado). The child participants' mean age was 47.3 months (range 36.0-62.0 months). Participants' mean age equivalence was approximately 36 months as determined by the Mullen visual reception (VR) subscale (VR is often used as a proxy for nonverbal cognitive ability). Eighty-five percent of the participants were White, $8 \%$ were Black, $5 \%$ were Asian, and $2 \%$ were multiracial. Trained research staff confirmed diagnosis of ASD with the Autism Diagnostic Observation Schedule (ADOS) (Lord, Rutter, DiLavore, \& Risi, 1999). Each child was enrolled in one of two comprehensive treatment model (CTM) programs in a public school (LEAP, 33 children, or TEACCH, 33 children) or in the control condition or BAU public school classroom (34 children). The term "BAU" refers to classrooms in which teachers do not adhere to one theoretical/conceptual framework to guide their instructional practices (e.g., applied behavior analysis); rather, teachers in these classrooms use a variety of intervention approaches from multiple models. This eclectic model is the one typically used with children with ASD in public schools (Stahmer, Collings, \& Palinkas, 2005). From the larger sample $(n=198)$, participants were randomly selected until we had obtained approximately equal numbers of participants per classroom type.

\section{Classrooms/Teachers}

All teachers and classrooms had to meet the following criteria to be included in the study: classrooms operated within the public school system, teachers licensed to teach in their respective states, and lead teacher having taught children with ASD for at least 2 years prior to inclusion in the study. In addition, TEACCH and LEAP teachers must have attended a formal TEACCH or LEAP training (at least at the district level) and have worked in a TEACCH or LEAP classroom for at least 2 years prior to the study.

The TEACCH and LEAP CTMs represent somewhat contrasting approaches to educating children with ASD. TEACCH bases its conceptual orientation in cognitive-social learning theory and subscribes to a "culture of autism," in which accommodations such as visual schedules and work systems (Hume, Plavnick, \& Odom, 2012) are made to the environment versus the individual to promote the child's engagement and learning (Mesibov, Shea, \& Schopler, 2005). In the context of schools, this has often manifested itself in children with ASD being educated together in classrooms that are separate from the children's typically developing peers. In contrast, LEAP bases its treatment approach on a blend of applied behavior analysis as well as common tenets of early childhood education (Strain \& Hoyson, 2000; Strain, Hoyson, \& Jamieson, 1985) with a goal of reducing children's characteristics of autism that interfere with their learning opportunities. The LEAP model uses an inclusive education approach whereby children with ASD are taught alongside typically developing, sameaged peer confederates. Irrespective of classroom type, all classrooms, including BAU classrooms, had to meet a priori determined screening criteria to ensure classroom quality. Specifically, all classrooms had to meet an "average" rating (score of three out of five) on four subscales of a validated classroom quality measure: the PDA program assessment (Professional Development in Autism Center, 2008) during an initial classroom visit. In addition, TEACCH and LEAP classrooms had to meet above-average ratings (3.5 out of 5 ) on model-specific subscales and items on their respective fidelity of implementation measures.

\section{Procedure}

Research staff collected 30-min videotaped samples for each child participant (i.e., focal child) during center time at pretest (beginning of the school year) and posttest (end of the school year). For the purposes of this study, only posttest video samples were used. Video lengths ranged from $24 \mathrm{~min}$ to $30 \mathrm{~min}$ as a result of unforeseen issues (e.g., child leaving the classroom unexpectedly during videotaping, issues with videotaping). Center time was selected as the observation context because it was a common feature across all classroom types. Adults, other children, or the focal child could select the activities the focal child engaged in during this time. Classroom activities commonly occurred 
in such areas as manipulatives, dramatic play, sensory, pre-academics, computers, large blocks, or art. Instructions were provided to research staff to video the focal child and the environmental context (i.e., film focal child, center where focal child was located, and other children/adults in center). Filming could begin during centers or another activity (e.g., transition or circle time), but no more than 5 min of an activity other than centers could be filmed.

Social behavior was operationally defined as any positive gestural/motor or verbal/vocal behavior directed toward either a peer or an adult. Examples of social behavior included talking to a peer/adult, touching an adult or peer (e.g., holding hands, hugging), and responding to an adult or peer (e.g., answering an adult's question or following directions). Negative social behavior was operationally defined as any negative gestural/motor or verbal/vocal behavior directed toward either a peer or an adult (e.g., hitting, saying "no"). Negative social behaviors were not coded or included in this study because of their low frequency of occurrence.

Social behavior was coded using MTS, PIR, and event coding. Initially, Procoder observational software (Tapp \& Walden, 2000) was used to code all videos using a MTS framework. An eco-behavioral coding scheme, the Code for Active Student Participation and Engagement (CASPER), was applied across child and adult behaviors as well as classroom environmental features to operationally define all behavioral codes (Tsao, Odom, \& Brown, 2001). Social behavior was coded using a mutually exclusive hierarchical coding system for the following social behaviors: social behavior directed to an adult, negative social behavior directed to an adult, social behavior directed to a peer, negative social behavior directed to a peer, social behavior directed from a peer to the focal child, negative social behavior directed from a peer to the focal child, no social behavior, and cannot tell. In other words, only one category of social behavior could be coded at a time with those listed first (e.g., social behavior directed to an adult) taking precedence if more than one social behavior occurred simultaneously. Social behavior was coded using MTS in 10-s intervals for a total of six possible social behaviors per minute.

Next, each video was coded simultaneously using PIR and event coding. For event coding, the total number of social behaviors directed toward peers and adults were tallied separately within each 10-s interval to assist with analyzing interobserver agreement (IOA). For PIR, social behaviors directed toward both peers and adults (separately) were coded as either occurrence or nonoccurrence for each 10-s interval for a total of six possible social behaviors per minute.

\section{Data Analysis}

In order to compare across the three coding methods, the total number of social behaviors directed to both peers and adults obtained for each method were converted to a rate per minute. To obtain the rate per minute of social behaviors, the total number of social behaviors for each coding method (i.e., MTS, PIR, and event) was divided by the length of videotaped sample. For example, if MTS captured 25 social behaviors, PIR 33 social behaviors, and event 41 social behaviors within a 28-min videotaped sample, the rate per minute for each would be an estimation of 0.89 social behaviors for MTS, an estimation of 1.18 social behaviors for PIR, and 1.46 social behaviors for event coding. Converting each frequency to a rate per minute allowed for a clear, unbiased comparison of each coding method across the classroom type. If frequency count was used rather than rate per minute, participants who had a full 30-min sample would have the opportunity for a greater weight in the analysis than a participant with only a 24-min sample. Further, in order to compare the three coding methods, we needed to use the same metric. For this analysis, we selected rate versus percentage of intervals to allow for a comparison across the three different coding methodologies. In order to increase the accuracy of using a rate conversion with MTS and PIR, we employed brief, 10 -s intervals. Given the low rates of social behavior, it is likely that MTS and PIR represent a close approximation to actual behavior rates. Previous research findings indicate that the use of shorter intervals with MTS and PIR will result in a more accurate representation of behavior (Ary \& Suen, 1983; Powell et al., 1975).

Pearson's correlations were used to determine the association between the coding methods. The strength of relationships were based on the following: $r$ values between 0 and 0.3 indicated weak positive relationships, 
TABLE 1

IOA across Models for Social Behavior Directed toward Adults and Peers

\begin{tabular}{|c|c|c|c|c|c|c|}
\hline & \multicolumn{2}{|c|}{ Momentary } & \multicolumn{2}{|c|}{ Interval } & \multicolumn{2}{|c|}{ Event } \\
\hline & kарра & $A /(A+D)$ & kарра & $\mathrm{A} /(\mathbf{A}+\mathbf{D})$ & kappa & $A /(A+D)$ \\
\hline Toward adults & $\begin{array}{c}0.68 \\
(0.21-1)\end{array}$ & $\begin{array}{c}0.98 \\
(0.95-1)\end{array}$ & $\begin{array}{c}0.96 \\
(0.85-1)\end{array}$ & $\begin{array}{c}0.94 \\
(0.81-1)\end{array}$ & $\begin{array}{c}0.93 \\
(0.83-1)\end{array}$ & $\begin{array}{c}0.91 \\
(0.83-1)\end{array}$ \\
\hline Toward peers & $\begin{array}{c}0.84 \\
(0.33-1)\end{array}$ & $\begin{array}{c}0.99 \\
(0.98-1)\end{array}$ & $\begin{array}{c}0.97 \\
(0.74-1)\end{array}$ & $\begin{array}{c}0.96 \\
(0.75-1)\end{array}$ & $\begin{array}{c}0.97 \\
(0.83-1)\end{array}$ & $\begin{array}{c}0.94 \\
(0.75-1)\end{array}$ \\
\hline
\end{tabular}

Note. $\mathrm{A}=$ agreement of occurrence, $\mathrm{D}=$ disagreement of nonoccurrence.

$r$ values between 0.3 to 0.7 indicated moderate positive relationships, and $r$ values between 0.7 and 1.0 indicated strong positive relationships (Ratner, 2009). To examine the differences of rate of social behavior per minute across models, ANOVAs were used. Eta-squared values are also reported. An etasquared criterion of 0.09 is a medium effect size for the social sciences (Pierce, Block, \& Aguinis, 2004). For all significant differences, post hoc tests using Tukey's honest significant difference were run to determine specific model differences.

\section{Results}

Two graduate-level research assistants were trained on the coding methods. Training initially included familiarization with the CASPER-III Training Manual for Observers (Tsao et al., 2001). For MTS, analysis and discussion followed practiced observations until trainers reached $80 \%$ agreement (i.e., the number of agreements divided by the number of agreements and disagreements) and a kappa of at least 0.80 for each variable. A similar training occurred for using PIR and event coding. Kappa was determined using Procoder observational software (Tapp \& Walden, 2000). Procoder was not used to determine agreement for PIR or event coding because Procoder counted agreements of no social behavior into calculations. To obtain a more accurate IOA, agreement for PIR and event coding was calculated in an Excel spreadsheet using the following formula: agreement/(agreement + disagreement). Twenty percent of videos were coded by both coders to examine the IOA. See Table 1 for IOA for each of the three coding methods. For event coding, IOA was calculated by occurrence of agreements within 10-s intervals. Therefore, if one observer coded two events in one interval and the other coded one, the observers had one agreement and one disagreement. The observers had the highest agreement (0.99) when coding social behavior toward peers using MTS. The observers had the least agreement (0.91) when using event coding to code social behavior toward adults.

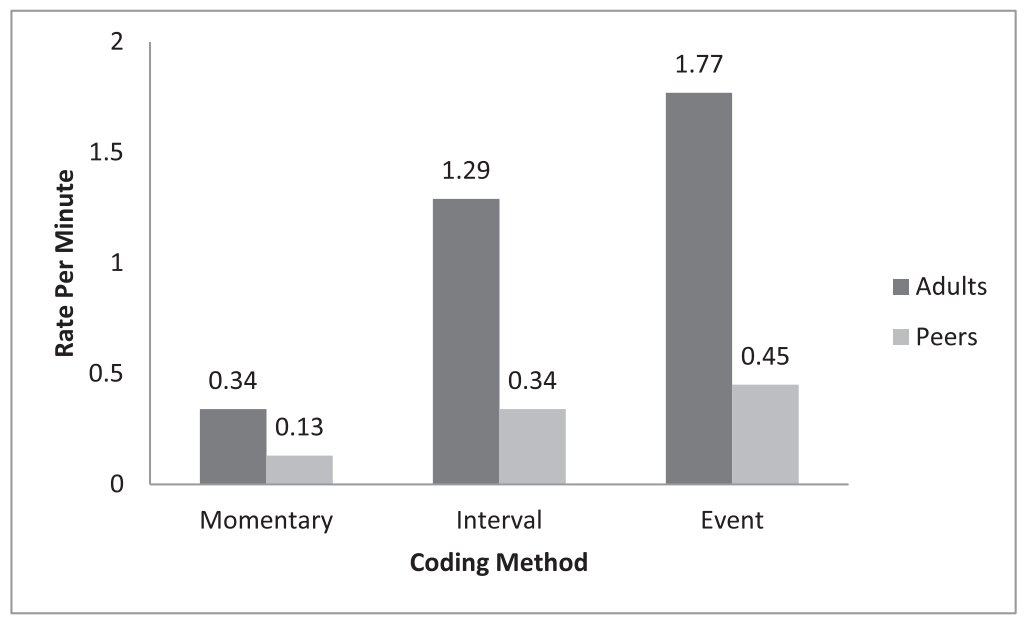

Figure 1. Social behavior. 
TABLE 2

Pearson's Correlation for Social Behavior Directed toward Adults

\begin{tabular}{|c|c|c|c|c|c|c|}
\hline & \multicolumn{2}{|c|}{ Momentary } & \multicolumn{2}{|c|}{ Interval } & \multicolumn{2}{|c|}{ Event } \\
\hline & Peer & Adult & Peer & Adult & Peer & Adult \\
\hline Momentary & 1.00 & 1.00 & $0.882^{\mathrm{a}}$ & $0.732^{\mathrm{a}}$ & $0.890^{\mathrm{a}}$ & $0.738^{a}$ \\
\hline Interval & & & 1.00 & 1.00 & $0.991^{\mathrm{a}}$ & $0.963^{\mathrm{a}}$ \\
\hline Event & & & & & 1.00 & 1.00 \\
\hline
\end{tabular}

aStrong positive linear correlation at the 0.001 level.

\section{Social Behavior toward Adults}

Event coding resulted in the highest number of social behaviors directed toward an adult with a mean of 1.77 (range 0.13-6.17) social behaviors directed toward adults per minute $(m=53.03$ total social behaviors in 30-min segment). PIR estimated 1.29 social behaviors (range 0.13-3.45) per minute ( $m=$ 38.66 total estimation of social behaviors in 30-min segment), followed by MTS with 0.34 (range $0-1.87$ ) estimated social behaviors per minute ( $m=10.23$ total estimation of social behaviors in 30-min segment; see Figure 1). All methods were strongly correlated with an $r$ value greater than 0.7 (see Table 2). Comparing all three coding methods, PIR to event had the strongest correlation with an $r$ of 0.963 ( $p$ $<.001)$. MTS and PIR had the weakest correlation of $r=0.732(p<.001)$. However, this $r$ value indicated a strong correlation using Ratner's guidelines (2009).

\section{Social Behavior toward Peers}

Figure 1 displays the social behavior of focal children toward peers. Similar to social behavior toward adults, event coding resulted in the most social behaviors toward peers per minute with a rate of 0.45 (range $0-4.03 ; m=$ 13.49 total social behaviors in 30-min segment). This was followed by PIR (rate $=0.34$ estimated social behaviors per minute, range $0-2.7 ; m=10.29$ total estimation of social behaviors in 30-min segment) and MTS (rate = 0.13 estimated social behaviors per minute, range $0-1.14 ; m=3.82$ total estimated social behaviors in 30-min segment). All correlations between the various coding methods were significant. The strongest correlation was between event and PIR $(r=0.991, p<$ $.001)$, followed by MTS and event $(r=$ $0.890, p<.001)$ and MTS and PIR $(r=$ 0.882, $p<.001$ ) (see Table 2).

\section{Observational Methods and Classroom Types}

\section{Social Behavior towards Adults}

Figure 2 displays the social behavior directed toward adults of focal children across the three models. Across the three models, children in LEAP classrooms exhibited higher

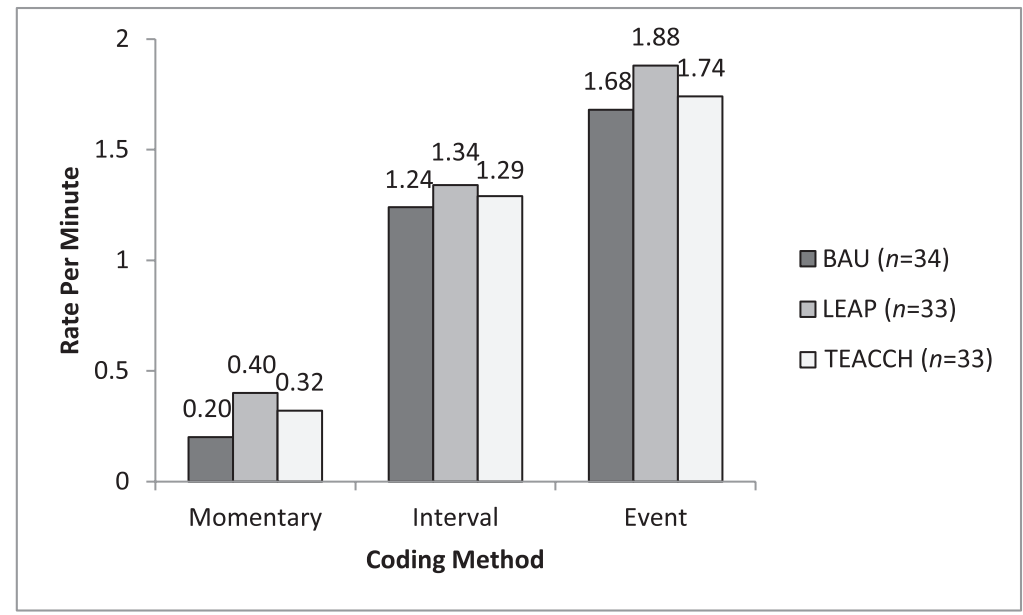

Figure 2. Social behavior directed toward adults across models. 


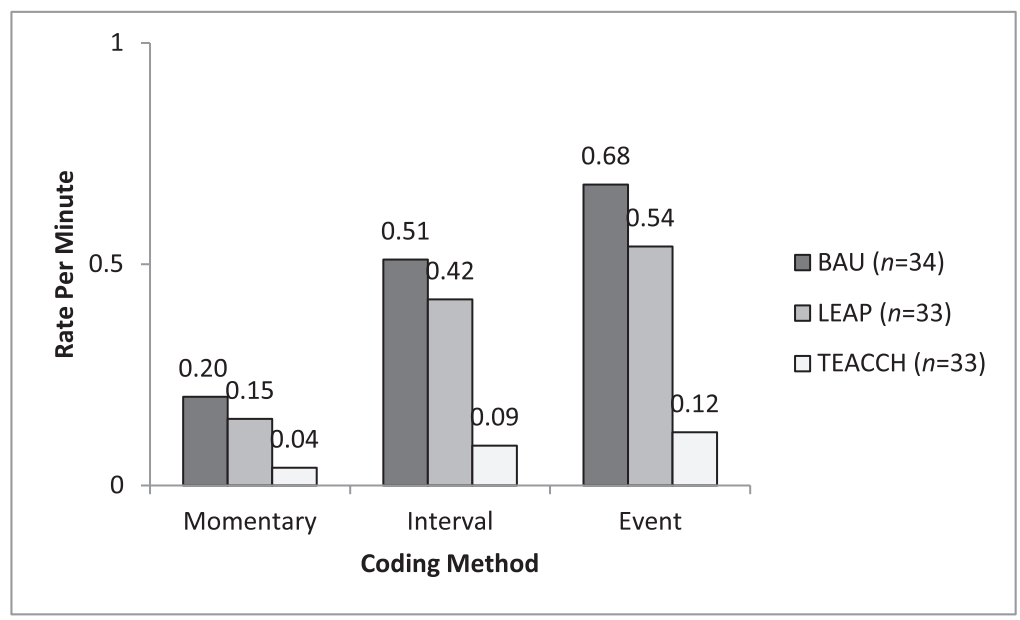

Figure 3. Social behavior directed towards peers across models.

rates of social behavior directed toward adults followed by students in TEACCH classrooms and then BAU classrooms. Analyses of variance were performed to determine if differences in social behavior existed between classroom programs (i.e., TEACCH, LEAP, and BAU) for each of the methods. No significant differences in social behavior directed toward adults were found.

\section{Social Behavior towards Peers}

The social behavior directed toward peers across the three models is depicted in Figure 3. Based upon the ANOVAs, all three coding methods resulted in significant group differences in social behavior directed toward peers. Further, a similar pattern emerged across the coding methods. Social behavior directed toward peers differed significantly $(p$ $=.008$ and $\left.\eta^{2}=0.10\right)$ when using MTS. Children in TEACCH classrooms were less likely $(p=.006)$ to display social behaviors toward peers than children in BAU classrooms. For PIR, social behavior directed toward peers again differed significantly $(p$ $=.001$ and $\eta^{2}=0.130$ ) with children in TEACCH classrooms displaying less social behavior than children in BAU $(p=.001)$ or LEAP ( $p=.018$ ) classrooms. Event coding also showed statistically significant differences between models $\left(p=.002\right.$ and $\eta^{2}=0.120$ ) with children in TEACCH classrooms less likely to display social behaviors toward peers than children in BAU $(p=.020)$ or $\operatorname{LEAP}(p=$ .028) classrooms.

\section{Discussion}

Confirming findings from previous research studies (Brown, Odom, Li, \& Zercher, 1999; Reszka et al., 2012; Tsao et al., 2008), social behaviors as assessed by all three coding methods for children with ASD were quite low. Mean social behaviors toward adults ranged from 0.34 to 1.77 behaviors per minute, depending on the coding method, and social behaviors toward peers ranged from 0.13 to 0.45 per minute. The findings indicated a strong relationship among MTS, PIR, and event coding for social behaviors directed toward both adults and peers. The relationship was strongest between PIR and event coding for social behaviors directed toward adults and peers. Further, the relationship among the coding methods was strongest for very lowoccurring behaviors (i.e., social behavior directed toward peers). Additionally, there was more variability between MTS and event coding as well as MTS and PIR. The variability was more evident as the frequency of social behaviors increased. In general, it appears that more social behaviors were more accurately coded when event coding and PIR methods were used compared with MTS.

In regards to the patterns of social behaviors for each coding method across classroom programs, results showed that a similar pattern emerged across the coding methods. While no statistical differences among CTMs were found for social behavior directed toward adults, visual inspection indicated a similar pattern. 
For all three coding methods, the most social behaviors directed toward adults occurred in LEAP classrooms followed by TEACCH and then BAU classrooms. The lack of significant differences for social behavior directed toward adults is perhaps not surprising given that no group differences were found in the larger study (see Boyd et al., 2014). One of the reasons could be that teachers in LEAP, $\mathrm{TEACCH}$, and BAU classrooms were using similar instructional strategies, such as visual supports, to educate and interact with children with ASD (Boyd et al., 2014).

Additionally, the coding methods produced similar patterns across models for social behavior directed toward peers. For PIR and event coding, significant differences were found among classroom types, with children in TEACCH classrooms less likely to display social behaviors toward peers than children in BAU and LEAP classrooms. Momentary time sampling only found statistically significant differences among children in TEACCH classrooms and children in BAU classrooms. However, visual inspection shows that children in TEACCH classrooms were less likely to demonstrate social behaviors toward peers than children in LEAP classrooms. Given that TEACCH classrooms were primarily comprised of children with ASD, fewer opportunities for peer social interactions might have been possible because a majority of the children likely had social deficits.

Overall, we found that the three coding methods produced similar patterns when used in different classroom types. The strong correlations found when examining social behavior toward adults and peers indicate that all three coding methods are viable options for capturing low-occurring social behaviors in young children with ASD. However, given the lower estimates for the occurrences of social behavior when using MTS, researchers should use caution when employing these methods and ensure the coding methodology accurately captures low-occurring behaviors.

\section{Limitations}

Several limitations need to be considered and addressed. First, videotaped observations are somewhat problematic. This method of coding may be especially limiting when using event coding or MTS. Children may be temporarily out of view, or the video may not show the social partner of the child. These errors in video recording can impact the number of behaviors observed. Additionally, recording individuals in a classroom may impact how adults and children interact.

This sample included only data from one time point, so no inferences can be made about the stability of social behavior over time or whether any group differences are maintained throughout the school year. Additionally, coders used the CASPER code hierarchy of social behaviors when employing MTS methodology; therefore, at each interval, participants could only be coded as directing social behavior toward an adult or peer. This allowed for the possibility of social behavior directed toward peers to not be captured. PIR and event coding allowed for simultaneous coding of both social behavior directed toward peers and adults. The three coding methodologies might have had a stronger relationship if the CASPER code allowed for simultaneous social behavior. Also, comparing the three coding methods and converting frequency data to rate data has been shown to be problematic (Repp et al., 1976; Yoder \& Symons, 2010). However, due to the various lengths of videotaped samples, data needed to be converted into a rate per minute in order to have a common dependent variable for comparison.

Further, this study does not address other behavioral assessment approaches that assess duration and sequential associations between behaviors but only examines behaviors without considering the context before or after a given behavior. Finally, the sample size is small, and perhaps different conclusions could be drawn if a larger sample size was used. However, this study is the first exploration comparing various observational coding methods for observing low-occurring social behaviors in children with autism.

\section{Implications}

These findings are intended to inform both researchers and practitioners as they carefully consider the selection and usage of behavior observation methods, specifically when coding low-incidence behaviors, such as social interaction between young children on the autism spectrum. Overall, the results confirm past research that MTS underrepresents lowfrequency behaviors when compared to event coding (Odom \& Ogawa, 1992). However, the strong relationship among the various coding methods suggests that using MTS as an 
approximation for low-occurring social behaviors is still a useful approach, in particular when reliability or costs prohibit other coding approaches. Further, if the research question addresses differences between conditions (e.g., focusing interventions like a peer-mediated approach versus a control condition or two comprehensive treatment models versus a control condition), it appears that MTS may be as sensitive to treatment conditions as is event coding or PIR. When costs and reliability issues are not a factor, use of PIR and event coding would result in the best reflection of actual behavior occurrence.

These findings may allow researchers and practitioners to weigh other potential benefits of sampling procedures in their decision making, such as reduced observer drift and fatigue, increased feasibility and likelihood of use by practitioners, and increased ease in obtaining and maintaining observer reliability. Although the literature is replete with articles and chapters that describe and use various observational methods (Harrop \& Daniels, 1986; Harrop et al., 1990; Odom \& Ogawa, 1992; Powell et al., 1975), few have empirically examined the association across procedures, and/or the relationships among them. These variables can and should contribute to decisions around the selection of direct behavioral assessment techniques.

\section{REFERENCES}

American Psychiatric Association. (2000). Diagnostic and statistical manual of mental disorders (DSM-IV-TR). Washington, DC: American Psychiatric Association.

Ary, D., \& Suen, H. K. (1983). The use of momentary time sampling to assess both frequency and duration of behavior. Journal of Behavioral Assessment, 5(2), 143-150.

Boyd, B. A., Hume, K., McBee, M. T., Alessandri, M., Gutierrez, A., Johnson, L., ... Odom, S. L. (2014). Comparative efficacy of LEAP, TEACCH, and non-model-specific special education programs for preschoolers with autism spectrum disorders. Journal of Autism and Developmental Disorders, 44, 336-380. doi: 10.1007/s10803013-1877-9

Brown, W., Odom, S., Li, S., \& Zercher, C. (1999). Ecobehavioral assessment in early childhood programs: A portrait of preschool inclusion. Journal of Special Education, 33, 138-153.

Conroy, M. A., Boyd, B. A., Asmus, J. M., \& Madera, D. (2007). A functional approach for ameliorating social skills deficits in young children with autism spectrum disorders. Infants and Young Children, 20, 242-254.
Cunningham, A. (2012). Measuring change in social interaction skills of young children with autism. Journal of Autism and Developmental Disorders, 42, 593-605. doi:10.1007/s10803-011-1280-3

Gardenier, N., MacDonald, R., \& Green, G. (2004). Comparison of direct observational methods for measuring stereotypic behavior in children with autism spectrum disorders. Research in Developmental Disabilities, 25,99-118. doi:10.1016/ j.ridd.2003.05.004

Hancock, T. B., \& Kaiser, A. P. (2002). The effects of trainer-implemented milieu teaching on the social communication of children with autism. Topics in Early Childhood Special Education, 22, 39-54.

Harrop, A., \& Daniels, M. (1986). Methods of time sampling: A reappraisal of momentary time sampling and partial interval recording. Journal of Applied Behavior Analysis, 19, 73-76.

Harrop, A., Daniels, M., \& Foulkes, C. (1990). The use of momentary time sampling and partial interval recording in behavioural research. Behavioural Psychotherapy, 18, 121-127.

Hume, K., Plavnick, J., \& Odom, S. (2012). Promoting task accuracy and independence in students with autism across educational setting through the use of individual work systems. Journal of Autism and Developmental Disorders, 42, 2084-2099. doi:10.1007/s10803-012-1457-4

Kearns, K., Edwards, R., \& Tingstrom, D. H. (1990). Accuracy of long momentary time-sampling intervals: Implications for classroom data collection. Journal of Psychoeducational Assessment, 8, 74-85.

Lord, C., Rutter, M., DiLavore, P., \& Risi, S. (1999). Autism Diagnostic Observation Schedule (ADOS). Los Angeles, CA: Western Psychological Services.

Mesibov, G. B., Shea, V., \& Schopler, E. (2005). The TEACCH approach to autism spectrum disorders. New York: Kluwer Academic/Plenum.

Odom, S., \& Ogawa, L. (1992). Direct observation of young children's social interaction with peers: A review of methodology. Behavioral Assessment, 14, 407-441.

Pierce, C. A., Block, R. A., \& Aguinis, H. (2004). Cautionary note on reporting eta-squared values from multifactor ANOVA designs. Educational and Psychological Measurement, 64, 916-924. doi:10.1177/0013164404264848

Powell, J., Martindale, A., \& Kulp, S. (1975). An evaluation of time-sample measures of behavior. Journal of Applied Behavior Analysis, 8, 463-469.

Professional Development in Autism Center. (2008). PDA Program Assessment. Seattle, WA: PDA Center at University of Washington.

Rapp, J. T., Colby-Dirksen, A. M., Michalski, D. N., Carroll, R. A., \& Lindenberg, A. M. (2008). Detecting changes in simulated events using partial-interval recording and momentary time sampling. Behavioral Interventions, 23, 237-269. doi:10.1002/bin.269 
Ratner, B. (2009). The correlation coefficient: Its values range between $+1 /-1$, or do they? Journal of Targeting, Measurement and Analysis for Marketing, 17, 139-142. doi:10.1057/jt.2009.5

Repp, A. C., Deitz, D. E., Boles, S. M., Deitz, S. M., \& Repp, C. F. (1976). Differences among common methods for calculating interobserver agreement. Journal of Applied Behavioral Analysis, 9, 109-113.

Reszka, S. S., Odom, S. L., \& Hume, K. A. (2012). Ecological features of preschools and the social engagement of children with autism. Journal of Early Intervention, 34, 40-56. doi:10.1177/ 1053815112452596

Rhine, R. J., \& Linville, A. K. (1980). Properties of onezero scores in observational studies of primate social behavior: The effect of assumptions on empirical analyses. Primates, 21, 111-122.

Stahmer, A., Collings, N., \& Palinkas, L. (2005). Early intervention practices for children with autism: Descriptors from community providers. Focus on Autism and Other Developmental Disabilities, 20, 66-79.

Strain, P. S., \& Hoyson, M. (2000). The need for longitudinal, intensive social skills intervention: LEAP follow-up outcomes for children with autism. Topics in Early Childhood Special Education, 20, 116-122.

Strain, P. S., Hoyson, M., \& Jamieson, B. (1985). Normally developing preschoolers as intervention agents for autistic-like children: Effects on class deportment and social interaction. Journal of the Division for Early Childhood, 9, 105-109.

Suen, H. K., \& Ary, D. (1989). Analyzing quantitative behavioral observational data. Hillsdale, NJ: L. Erlbaum.

Tapp, J., \& Walden, T. A. (2000). PROCODER: A system for collection and analysis of observational data from videotape. In T. Thompson, D. Felece, \& F. J. Symons (Eds.), Behavioral observation: Technology and applications in developmental disabilities (pp. 61-70). Baltimore, MD: Paul H. Brookes Publishing Co.

Thompson, T., Symons, F. J., \& Felce, D. (2000). Principles of behavioral observation: Assumptions and strategies. In T. Thompson, D. Felce, \& F. J. Symons (Eds.), Behavioral observation:
Technology and applications in developmental disabilities (pp. 3-16). Baltimore, MD: Brookes.

Tsao, L., Odom, S. L., \& Brown, W. B. (2001). Code for active student participation and engagementRevised (CASPER III): A training manual for observers. Bloomington, IN: Indiana University.

Tsao, L., Odom, S. L., Buysse, V., Skinner, M., West, T., \& Vitztum-Koamecki, J. (2008). Social participation of children with disabilities in inclusive preschool programs: Program typology and ecological features. Exceptionality, 16, 125-140. doi:10.1080/09362830802198203

Wong, C., \& Kasari, C. (2012). Play and joint attention of children with autism in the preschool special education classroom. Journal of Autism and Developmental Disorders, 42, 2152-2161. doi:10.1007/s10803-012-1467-2

Yoder, P., \& Stone, W. (2006). Randomized comparison of two communication interventions for preschoolers with autism spectrum disorders. Journal of Consulting and Clinical Psychology, 74, 426-435. doi:10.1037/0022-006X.74.3.426

Yoder, P. J., \& Symons, F. J. (2010). Observational measurement of behavior. New York: Springer Pub.

\section{AUTHOR'S NOTE}

Development of this paper was partially supported with funding from the Institute of Education Sciences, U.S. Department of Education (R324B070219). The opinions expressed by the authors are not necessarily reflective of the position, or endorsed by, the U.S. Department of Education.

Address correspondence to Ann Sam, 3C Institute, 1901 North Harrison Ave., Suite 200, Cary, NC 27513; Email:sam@3cisd.com.

MANUSCRIPT

Initial Acceptance: 5/11/14

Final Acceptance: 6/13/14 